\title{
On the breakup of viscous liquid threads
}

\author{
Demetrios T. Papageorgiou* \\ Department of Mathematics and \\ Center for Applied Mathematics and Statistics \\ New Jersey Institute of Technology \\ Newark, New Jersey 07102
}

\begin{abstract}
A one-dimensional model evolution equation is used to describe the nonlinear dynamics that can lead to the breakup of a cylindrical thread of Newtonian fluid when capillary forces drive the motion. The model is derived from the Stokes equations by use of rational asymptotic expansions and under a slender jet approximation. The equations are solved numerically and the jet radius is found to vanish after a finite time yielding breakup. The slender jet approximation is valid throughout the evolution leading to pinching. The model admits self-similar pinching solutions which yield symmetric shapes at breakup. These solutions are shown to be the ones selected by the initial boundary value problem, for general initial conditions. Further more, the terminal state of the model equation is shown to be identical to that predicted by a theory which looks for singular pinching solutions directly from the Stokes equations without invoking the slender jet approximation throughout the evolution. It is shown quantitatively, therefore, that the one-dimensional model gives a consistent terminal state with the jet shape being locally symmetric at breakup. The asymptotic expansion scheme is also extended to include unsteady and inertial forces in the momentum equations to derive an evolution system modelling the breakup of Navier-Stokes jets. The model is employed in extensive simulations to compute breakup times for different initial conditions; satellite drop formation is also supported by the model and the dependence of satellite drop volumes on initial conditions is studied.
\end{abstract}

* This work was supported by the Air Force Office for Scientific Research (Grant F49620-94-1-0242) and by the National Science Foundation (Grant DMS 9-9-0070). Partial support was also provided by the National Aeronautics and Space Administration under NASA Contract No. NAS1-19480 while the author was in residence at the Institute for Computer Applications in Science and Engineering (ICASE), NASA Langley Research Center, Hampton, VA 23681-0001. 


\section{Introduction}

It is well known that a perfectly cylindrical jet of liquid which supports surface tension at its free interface is an unstable stationary solution of the equations of motion (both viscous and inviscid) - any uniform axial velocity can be removed by a Galilean transformation, so we concentrate on stationary unperturbed jets. The instability is driven by capillary forces: If the radius of the jet decreases locally at some axial position, capillary forces induce a local increase in pressure (the pressure outside the jet is constant here) and conversely a local increase in radius causes a local decrease in pressure just below the interface; fluid will flow from regions of interfacial depression to regions of interfacial expansion and by mass conservation the depression/expansion will decrease/increase. Linear stability analyses based on normal modes have been carried out by Rayleigh for both inviscid (see [1]) and viscous (see [2]) jets with the effect of the surrounding fluid neglected. The effect of a viscous surrounding phase has been included in the linear stability analysis of Tomotika [3]. The viscous dispersion relation given in [2] is valid in the limit of a highly viscous fluid; the general dispersion relation for arbitrary viscosities is given in [3] as well as Chandrasekhar ([4], p. 541). The present work considers Stokes flows and so Rayleigh's dispersion is useful, then. Following Tomotika, normal mode disturbances are considered proportional to $\exp (i(n t+k z))$, where $t$ is time, $z$ is axial distance, $k$ is the wavenumber of the disturbances and in their growth rate: Solution of the linear eigenvalue problem in the case of Stokes jets with the surrounding phase neglected, yields the following dimensional growth rates (see [3], $[4])$ :

$$
\text { in }=\frac{\sigma}{2 R \mu} \frac{k^{2} R^{2}-1}{k^{2} R^{2}+1-k^{2} R^{2} I_{0}^{2}(k R) / I_{1}^{2}(k R)},
$$

where $\sigma$ is the surface tension coefficient (assumed to be constant), $\mu$ is the fluid viscosity and $R$ is the unperturbed jet radius. According to (1), the most unstable wave has $k=0$ and the growth rate is then given by

$$
i n_{0}=\frac{\sigma}{6 R \mu} .
$$

This long-wave instability result is due to the fact that time derivatives are dropped in the momentum equations. Its inclusion (or inclusion of a surrounding phase) provides a dispersion relation with a non-zero maximally growing wavenumber - see Tomotika's results. Our interest in this article is to generate analytical nonlinear structures which coincide with boundary integral numerical solutions, for example, in the long wave regime and in particular near pinching. The theory presented in this article, then, is a nonlinear long wave one and so on linearization of the obtained evolution equations, the growth rates (2) emerge (see below). It is worth noting that since the maximum growth rate is for infinitely long waves (as predicted theoretically by linear theory at least), a long wave nonlinear ansatz is justified. For inviscid and viscous (but not Stokes) jets the maximum growth rate occurs at a finite wavenumber which lies between 0 and 1 (in non-dimensional terms) and long wave theories, then, may not be consistent with the linear results; they can, however, describe phenomena such as jet pinching, which are beyond the scope of linear theory (see later).

Experimental observations of jet breakup phenomena have been carried out by Chaudhary and Maxworthy [5], [6], Donelly and Glaberson [7], Goedde and Yuen [8] and Peregrine, 
Shoker and Symon [9]; the latter investigation considers breakup with gravity being important also. More recently Tjahjadi, Stone and Ottino [10] have carried out an experimental study with highly viscous fluids using a Couette device; numerical boundary integral simulations based on the Stokes equations are also described and compared to the experiments. This work is an extension of previous experiments and computations by Stone and Leal [11] who consider the breakup of an initially extended drop. Simulations based on the NavierStokes equations and different Reynolds numbers (covering inviscid to viscous flows) have recently been applied by Richards, Lenhoff and Berris [12] to describe the motion of a viscous jet injected into another viscous liquid. Comparisons of the simulated breakup with experiments is made with good overall agreement. All the simulations described are carried out for axisymmetric jets; this assumption is consistent with linear theory as well as many experimental situations especially if the Reynolds number is not too large. For a review of drop formation in circular jets, see Bogy [13].

Our interest is in the description of the breakup from the viewpoint that it is a singularity of the equations of motion with the jet radius vanishing after a finite time at some axial location. The theory developed is a local one and the singular time as well as the axial position where breakup first occurs, depend on initial conditions. The main reason why such local structures are desirable analytically is that they provide a rational way of continuing the solutions beyond breakup after the topology changes. In fact simple mass and momentum balances can provide regular initial conditions for the dynamics beyond breakup by assuming that a spherical blob of fluid is attached to the end of the breakaway jet (see Ting and Keller [14] for inviscid flows and Papageorgiou [15] for Stokes and Navier-Stokes jet flows). Our approach is based on working with a simplified set of evolution equations which arise from the governing equations after a slender jet ansatz is adopted. One-dimensional slender jet models have recently been used by many authors in the modeling of viscous liquid jets (see for example Renardy [16], Eggers and Dupont [17], Eggers [18], [19], and Garcia and Castellanos [20]). Such approaches are unsteady extensions of the steady fiber extrusion problem considered by previous investigators (see for example Schultz and Davis [21] and references therein, as well as the review article by Denn [22]). In the present work we mostly consider breakup governed by the Stokes equations (extensions which include inertail and unsteady terms in the momentum equations are also derived); this is a problem with applications in microgravity flows, for instance, where fluid viscosities are high and typical Reynolds numbers are small. A system of one-dimensional partial differential equations is derived by assuming that the ratio between the maximum jet radius to the wavelength of interfacial deflections is an asymptotically small parameter which can be used in an asymptotic expansion to capture the leading order evolution. (Note that higher order corrections can be calculated routinely within this framework). These equations have been derived previously in [16] using a Lagrangian coordinate system. Renardy also proves a theorem which states that in the Newtonian case these equations have solutions with the radius vanishing after a finite time (he also shows that this is not the case for several viscoelastic models). The equations support similarity solutions corresponding to pinching and we show that these are identical to pinching solutions obtained directly from the full Stokes equations (see also [15] where the Navier-Stokes system is also studied). Analysis of the similarity equations fixes universal scaling laws for the breakup and we confirm these scaling exponents by direct numerical solution of the model equations for different initial conditions. 
The article is organized as follows. In Section 2 the equations together with interfacial boundary conditions are given along with the non-dimensionalization based on capillary scales. Section 3 derives the slender jet model comprised of a system of coupled nonlinear evolution equations. A generalization of the expansions to include inertial and unsteady momentum effects is given in an Appendix. In Section 4 we construct self-similar solutions valid as the jet pinches after a finite time. This is done for (i) the evolution equations derived in Section 2, and, (ii) by looking for singular solutions directly from the full Stokes equations given in Section 2. The similarity equations are the same and they are solved to obtain the solutions in closed implicit form. The scaling exponents are also determined and are shown to be universal. Section 5 is devoted to the numerical solution of the evolution equations. It is shown that the analytical self-similar solutions of Section 4 are the ones obtained at pinching. This is done by comparison of scaling exponents and functions provided by the simulation with the analytical predictions. Both symmetric and non-symmetric initial conditions (for the jet shape) are used and the analytical results are verified in both cases.

\section{Governing equations}

Consider the evolution of a viscous cylindrical column of fluid of viscosity $\mu$. Initially the jet is a perfect infinite cylinder of radius $R$ and zero velocity (a constant axial velocity can be removed by a suitable Galilean transformation); this is an exact solution of the equations of motion and boundary conditions and is the flow used in linear stability theories in the calculation of the initial stages of the instability. Even though linear theory cannot provide a quantitative description of the flow at breakup, it does give useful insight into the competing physical mechanisms acting. It is found, then, that capillary forces drive the instability which leads to pinching (see Introduction). The pertinent scales are: lengths scale with $R$; velocities scale with $\frac{\sigma}{\mu}$; pressure scales with $\frac{\sigma}{R}$; time scales with $\frac{\mu R}{\sigma}$. The equations are made dimensionless by introduction of the variables

$$
(r, z)=R(\bar{r}, \bar{z}), \quad(u, w)=\frac{\sigma}{\mu}(\bar{u}, \bar{w}), \quad p=\frac{\sigma}{R} \bar{p}, \quad t=\frac{\mu R}{\sigma} \bar{t} .
$$

Substitution of (3) into the Stokes equations and interfacial boundary conditions and dropping of the bars, yields the following non-dimensional system:

$$
\begin{aligned}
\triangle u-\frac{1}{r^{2}} u & =p_{r}, \\
\triangle w & =p_{z}, \\
\frac{1}{r}(r u)_{r}+w_{z} & =0,
\end{aligned}
$$

where $\triangle \equiv \frac{\partial^{2}}{\partial r^{2}}+\frac{1}{r} \frac{\partial}{\partial r}+\frac{\partial^{2}}{\partial z^{2}}$. The interfacial conditions of tangential stress balance, normal stress balance and kinematic condition, on $r=S(t, z)$ are

$$
\begin{array}{r}
\left(u_{z}+w_{r}\right)\left(1-S_{z}^{2}\right)+2 u_{r} S_{z}-2 w_{z} S_{z}=0 \\
p-2 u_{r}-\left(-p+2 w_{z}\right) S_{z}^{2}+2\left(u_{z}+w_{r}\right) S_{z}=-\left(S_{z z}-\frac{1}{S}\left(1+S_{z}^{2}\right)\right)\left(1+S_{z}^{2}\right)^{-1 / 2} \\
u=S_{t}+w S_{z}
\end{array}
$$


In addition to the interfacial conditions (8)-(10) we impose regularity of flow quantities on the jet axis $r=0$.

We are interested in solutions of the system (4)-(10) in the strongly nonlinear regime and in particular when pinching occurs and the jet radius tends to zero at some axial position. Direct numerical solutions have been given in [10] and [11] by use of boundary integral techniques. Here we propose a quantitative description of the pinching by use of an asymptotic theory.

\section{$3 \quad$ The evolution equations governing pinching}

The evolution equations arise from a nonlinear long wave theory. If we assume that a typical dimensional length scale in the axial direction is $D$, then the ratio $\epsilon=\frac{R}{D}$ is taken to be a small quantity and we seek a leading order solution of an asymptotic expansion in $\epsilon$. Such approximations have been used extensively in the description of nonlinear long wave interfacial flows (see Introduction). At first sight the theory seems arbitrary since the parameter $D$ does not have a distinct physical meaning. The usefulness of the evolution equations given below along with their solutions lies in the fact that as a pinch forms the radial length scale is at most of the order of the axial length scale (i.e. the axial extent of the pinch region); if the radial length scale is asymptotically smaller than the axial one, a long wave approximation is valid to leading order and so the model equations describe local pinching solutions of the full equations even though the transient motion may be inaccurate. A theory which constructs pinching solutions of the Stokes equations directly, has been given in $([15])$. It is shown later that the two theories are in complete agreement thus lending weight to the relatively simple model equation approach.

The long wave ansatz is easily applied to (4)-(10) by introducing the transformation $\frac{\partial}{\partial z} \rightarrow \epsilon \frac{\partial}{\partial z}$; in addition the appropriate expansions proceed in powers of $\epsilon^{2}$ (this can be seen from the streamfunction formulation of the Stokes equations in cylindrical coordinates which yields the biharmonic equation for the streamfunction implying that the velocities expand in powers of $\epsilon^{2}$ ):

$$
\begin{gathered}
u(t, r, z)=u_{0}+\epsilon^{2} u_{1}+\ldots, \\
w(t, r, z)=\frac{1}{\epsilon} w_{0}+\epsilon w_{1}+\ldots, \\
p(t, r, z)=p_{0}+\epsilon^{2} p_{1}+\ldots, \\
S(t, z)=S_{0}+\epsilon^{2} S_{1}+\ldots .
\end{gathered}
$$

Substitution of (11)-(14) into the governing equations and boundary conditions gives a sequence of problems. The leading order velocity components are found from (5) and (6) and are

$$
w_{0} \equiv w_{0}(t, z), \quad u_{0}=-\frac{1}{2} r w_{0 z}
$$

Substitution of this expression for $u_{0}$ into (4) gives $p_{0 r}=0$ and so $p_{0} \equiv p_{0}(t, z)$. This readily yields the correction $w_{1}$ from (5) as well as the leading order pressure distribution throughout the jet from the normal stress balance condition (9); the continuity equation then gives $u_{1}$ in 
terms of $w_{0}$ and the process can be continued to higher order. The results which are needed for the evolution equation are:

$$
w_{1}(t, r, z)=-\frac{1}{4} r^{2}\left(w_{0 z z}-p_{0 z}\right)+A(t, z), \quad p_{0}(t, z)=\frac{1}{S_{0}}-w_{0 z}
$$

where $A(t, z)$ is to be determined but it does not enter the leading order dynamics. With these solutions available, the evolution equations are obtainable from the tangential stress balance condition (8) and the kinematic condition (10). To leading order the tangential stress balance gives $w_{0 r}=0$ which is already satisfied by (15). Next at order $\epsilon$ and at order one in (8) and (10) respectively, we find

$$
\begin{aligned}
u_{0 z}+w_{1 r}+2 u_{0 r} S_{0 z}-2 w_{0 z} S_{0 z} & =0, \quad r=S_{0}(t, z), \\
u_{0} & =S_{0 t}+w_{0} S_{0 z} .
\end{aligned}
$$

The desired evolution equations are obtained from (17) and (18) by elimination of $u_{0}$ and $w_{1}$ in favor of $w_{0}$ alone to yield an evolution system for $S_{0}$ and the leading order axial velocity $w_{0}$. This system is:

$$
\begin{aligned}
S_{0 t}+w_{0} S_{0 z}+\frac{1}{2} w_{0 z} S_{0} & =0 \\
\frac{3}{2} S w_{0 z}+3 S_{0 z} w_{0 z}+\frac{S_{0 z}}{2 S_{0}} & =0
\end{aligned}
$$

Asymptotic and numerical solutions of (19) and (20) are given in later Sections but some comments on the physical origin of these equations are useful.

An integrated form of the evolution equations has been derived previously by M. Renardy [16] by use of physical arguments. The first equation describes conservation of mass for slender jets which is most easily seen by multiplication of (19) by $S_{0}$ to yield the conservation form

$$
\left(S_{0}^{2}\right)_{t}+\left(w_{0} S_{0}^{2}\right)_{z}=0
$$

The second equation was written down by Renardy by considering the force acting on a cross-section of the slender jet. The Stokes flow has no inertia and so this force must be constant along the jet; equation (20) is the $z$-derivative of this force. This is most easily seen by multiplication of $(20)$ by $S_{0}$ and writing it in the form

$$
\left(3 S_{0}^{2} w_{0 z}+S_{0}\right)_{z}=0
$$

These ideas have also been used in [15] in following jet evolution just beyond pinching by description of overall mass and momentum balance equations (see also [14] for an application to inviscid flows).

We note also that a simple modification of (11)-(14) that allows for non-zero Reynolds numbers and introduces inertial and unsteadiness into the momentum equations leads to a set of evolution equations which can be used to model pinching of jets governed by the Navier-Stokes equations. This analysis is included in Appendix A. 


\section{Finite-time singularities. Self-similar solutions.}

The evolution equations (19) and (20) have been proven by Renardy [16] to possess singularities with the jet radius vanishing after a finite time. A Langrangian formulation was used to prove the theorem and in particular it is established that arbitrarily small initial conditions can lead to breakup. Our interest is in the related problem of quantifying such terminal states and in particular in establishing any type of dominant solutions at breakup. To this end we employ numerical computations of the initial value problem and a local analysis of breakup by construction of self-similar solutions. In what follows we address the description of local structures at breakup. This is done for (i) the long wave evolution system derived above, and, (ii) directly from the full Stokes equations. The latter analysis has been given in [15] where unique scaling laws were established by solution of a nonlinear eigenvalue problem (see later). In what follows we show that the similarity solutions of the model equations are identical to those found by a direct analysis of the Stokes system and we test the analytical self-similar structures and in particular the unique scaling exponents, with numerical solutions obtained by solving the initial value problem (19) and (20). This is done in a later Section with excellent agreement.

\subsection{Pinching solutions of the model equations}

We work with (19) and (20) and assume that the jet radius vanishes after a finite time, $t_{s}$ say. The objective is to describe the solutions near this time, so that $\tau=t_{s}-t$ with $0<\tau<<1$, and near the axial position where the jet breaks (without loss of generality this position is taken to be the origin). A balance of terms in (19), (20) gives the order-of-magnitude estimates

$$
\frac{S_{0}}{\tau} \sim \frac{w_{0} S_{0}}{z}, \quad \frac{S_{0}^{2} w_{0}}{z} \sim S_{0}
$$

from which it follows that $S_{0} \sim \tau$ while $w_{0} \sim \frac{z}{\tau}$. Assuming that the phenomenon is a focussing one we can introduce a positive parameter $\beta$ which controls the extent of the similarity region by $z \sim \tau^{\beta}$. Formally, then, we look for pinching solutions of (19) and (20) in the form

$$
S_{0}(t, z)=\tau f(\xi), \quad w_{0}(t, z)=\tau^{\beta-1} g(\xi), \quad \xi=\frac{z}{\tau^{\beta}} \quad\left(\tau=t_{s}-t\right) .
$$

Note that the forms (23) above are an exact self-similar transformation since all terms in the governing equations are in balance. The situation is slightly different for the full Stokes equations (see later). Partial derivatives transform according to

$$
\frac{\partial}{\partial t} \rightarrow-\frac{\partial}{\partial \tau}+\frac{\beta \xi}{\tau} \frac{\partial}{\partial \xi}, \quad \frac{\partial}{\partial z} \rightarrow \frac{1}{\tau^{\beta}} \frac{\partial}{\partial \xi}
$$

Substitution of (23) and (24) into (19) and (20) gives the following equations for the scaling functions:

$$
\begin{aligned}
(g+\beta \xi) f^{\prime}+\left(\frac{1}{2} g^{\prime}-1\right) f & =0 \\
\frac{d}{d \xi}\left(3 f^{2} g^{\prime}+f\right) & =0 .
\end{aligned}
$$


Equation (26) can be integrated once to give

$$
g^{\prime}=-\frac{1}{3 f}+\frac{k}{f^{2}}
$$

where $k$ is a constant of integration. Equations $(25),(27)$ need to be solved and the constants $\beta$ and $k$ determined. In fact the value of $k$ can be expressed in terms of $f$ as explained next. The force balance equation (22) can be integrated in $z$ to yield (44) below and the function $\lambda(t)$ given by (45). ¿From the ansatz (23) and (44) it is clear that $\lambda \sim \tau$ as $\tau \rightarrow 0$ and in fact $\lambda=3 k \tau+\ldots$ then with $k$ as used in (27) above. The following expression for $k$ then follows,

$$
k=\frac{1}{3} \frac{\int_{-\infty}^{\infty}(1 / f) d \xi}{\int_{-\infty}^{\infty}\left(1 / f^{2}\right) d \xi},
$$

from either of two equivalent ways: (i) by integration of (27) over the range of $\xi$ and using the fact that axial velocities are zero far from the pinch (this is shown asymptotically later), (ii) by introduction of the ansatz (23) into the expression (45) for $\lambda$. This identification of $k$ is essential in the determination of a unique value of $\beta$ described later. Before doing this we present the construction of singular solutions of the full Stokes equations which yield an identical result.

\subsection{Pinching solutions of the Stokes equations.}

In this section we summarize results described in more detail in [15]. The idea is to construct solutions to (4)-(10) with the jet radius going to zero after a finite time. The system is not one dimensional in space as for the model equations and a similarity variable in the radial direction is also needed. The following transformations are appropriate

$$
\begin{array}{rr}
r=\tau^{\alpha} y, \quad z=\tau^{\beta} \xi, \quad S=\tau^{\alpha} f(\xi), \\
w=\tau^{\gamma} W(t, y, \xi), \quad u=\tau^{\gamma+\alpha-\beta} U(t, y, \xi), \quad p=\tau^{-\alpha} P,
\end{array}
$$

where $u$ follows from the continuity equation and $\alpha>\beta$ in keeping with slender geometries at breakup. The constants $\alpha, \beta, \gamma$ are to be determined along with the scaling functions (the use of the same symbols as before should not be confusing). Unlike the model equations, the transformations (30) do not retain all terms in the Stokes equations and an asymptotic expansion in powers of $\tau^{2 \alpha-2 \beta}$ is appropriate (this comes from the biharmonic operator for the streamfunction in much the same way as the $\epsilon^{2}$ expansion was established for the derivation of the model system). These expansions are

$$
w=\tau^{\gamma}\left(g(\xi)+\tau^{2 \alpha-2 \beta} W_{1}+\ldots\right), \quad u=\tau^{\gamma+\alpha-\beta}\left(-\frac{1}{2} y g_{0 \xi}+\tau^{2 \alpha-2 \beta} U_{1}+\ldots\right),
$$

which on substitution into the $z$-momentum equation (5) and balance of leading order terms yields

$$
\gamma=\beta-\alpha, \quad W_{1 y}=\frac{1}{2} y\left(P_{\xi}-w_{0 \xi \xi}\right)
$$


This in turn leads to $U_{1}$ and the result that $P_{y}=0$. Substitution of leading order solutions into the tangential stress balance equation (8) and the normal stress balance (9) give at $O\left(\tau^{-\beta}\right)$ and $O\left(\tau^{-\alpha}\right)$ respectively

$$
\left(f^{3} g^{\prime}\right)^{\prime}=\frac{1}{2} f^{3} P^{\prime}, \quad P=-g^{\prime}+\frac{1}{f} \quad \text { on } y=f(\xi)
$$

which can be integrated once to yield

$$
g^{\prime}=-\frac{1}{3 f}+\frac{k}{f^{2}},
$$

with $k$ a constant to be determined. A second equation and the determination of $\alpha$ comes from a leading order balance in the kinematic condition (10), which gives

$$
\alpha=1, \quad(g+\beta \xi) f^{\prime}+\left(\frac{1}{2} g^{\prime}-1\right) f=0 .
$$

It can be seen that the leading order similarity solutions found here are identical to those predicted by the long wave model system. Solutions which are to be compared with the numerical solutions of the evolution equations are described next.

\subsection{Solution of the similarity equations.}

To fix matters we work with equations (25) and (26). Solutions must be obtained for $-\infty<$ $\xi<\infty$. The behavior for large $\xi$ is easily established from the equations to be

$$
f(\xi) \sim|\xi|^{\frac{1}{\beta}}, \quad g(\xi) \sim|\xi|^{-\frac{1-\beta}{\beta}} \quad \text { as }|\xi| \rightarrow \infty .
$$

This asymptotic behavior can also be deduced from the similarity transformations (23) since far away from the pinch region (i.e. as $|\xi| \rightarrow \infty$ ) the solutions are expected to be independent of $t$; it follows, then, that $f(\xi) \sim|\xi|^{\frac{1}{\beta}}$ and $g(\xi) \sim|\xi|^{-\frac{1-\beta}{\beta}}$ which make $S_{0} \sim|z|^{\frac{1}{\beta}}$ and $w_{0} \sim|z|^{-\frac{1-\beta}{\beta}}$ which are the outer solutions as $|z| \rightarrow 0$. It follows from (34) that $g$ vanishes as $|\xi|$ tends to infinity which in turn implies that there is a point, $\xi_{0}$ say, where $g\left(\xi_{0}\right)+\beta \xi_{0}=0$. Such a point is a removable singular point of equation (33) and requires a local analysis; $\xi_{0}$ can be shifted to the origin by use of the transformations

$$
f \rightarrow f, \quad G(\eta)=g+\beta \xi_{0} \quad \eta=\xi-\xi_{0} .
$$

The point $\eta=0$, then, is a removable singularity if

$$
G(0)=0, \quad G^{\prime}(0)=2 .
$$

This is a condition required by smoothness of solutions. A local analysis for $|\eta|<<1$ gives

$$
f(\eta)=f_{0}+\eta^{2} f_{2}+\eta^{4} f_{4}+\ldots, \quad G(\eta)=2 \eta+\eta^{3} g_{3}+\ldots,
$$

where

$$
f_{0}=\frac{1}{12(1+\beta)}, \quad k=\frac{3+2 \beta}{72(1+\beta)^{2}},
$$


with the remaining coefficients expressible in terms of a single parameter $f_{2}$.

To get a closed form solution defined implicitly it is useful to eliminate $g(\xi)$ between (25) and (27), for example, to obtain

$$
f^{\prime \prime}=\frac{\left(f^{\prime}\right)^{2}}{f} \frac{(1-\beta) f^{2}+\frac{1}{3} f-\frac{k}{2}}{\left(f+\frac{3+2 \beta}{12(1+\beta)}\right)\left(f-f_{0}\right)},
$$

which can be integrated once to give

$$
\int_{f_{0}}^{f} \frac{\left(f+\frac{3+2 \beta}{12(1+\beta)}\right)^{\beta+\frac{1}{2}}}{f\left(f-f_{0}\right)^{1 / 2}} d f=A \eta .
$$

The substitution

$$
f=\frac{1}{12(1+\beta)} \cosh ^{2}(\theta),
$$

in (37) above, leads to the implicit solution

$$
\begin{aligned}
f(\eta) & =\frac{1}{12(1+\beta)} \cosh ^{2}(\theta), \\
\frac{1}{(12(1+\beta))^{\beta}} \int_{0}^{\theta} \frac{\left(\cosh ^{2} \theta+3+2 \beta\right)^{\beta+\frac{1}{2}}}{\cosh \theta} d \theta & = \pm A \eta, \\
G(\eta) & =\int_{-\infty}^{\eta}\left(-\frac{1}{3 f}+\frac{k}{f^{2}}\right) d \eta+\beta \xi_{0},
\end{aligned}
$$

where

$$
A=\frac{24}{(1+\beta)}\left(\frac{2+\beta}{6(1+\beta)}\right)^{\beta+\frac{1}{2}} \sqrt{f_{2}},
$$

and \pm corresponds to $\eta$ positive and negative respectively. Using the boundary conditions $G(-\infty)=G(+\infty)=\beta \xi_{0}$ we see that

$$
k=\frac{1}{3} \frac{\int_{0}^{\infty}(1 / f) d \eta}{\int_{0}^{\infty}\left(1 / f^{2}\right) d \eta}
$$

which is seen to be identical to (28) once we note the symmetry of $f$. A numerical procedure is required to find admissible values of $\beta$. The first thing to note is that the ratio of the two integrals in (39) is independent of $f_{2}$ as can be seen by a rescaling of $\eta$ in (38) (this has been established numerically also). Given a value of the scaling parameter $\beta$, the solution $f(\eta)$ is constructed from (38) by prescribing a value of $\theta$ which implies an elevation $f$ at an axial position $\eta$ determined by integrating (38) over the appropriate $\theta$-range; with $f(\eta)$ known over an interval $0 \leq \eta \leq \eta_{\max }$, where $\eta_{\max }$ is sufficiently large for the asymptotic behavior (34) to be valid, an iteration in $\beta$ is carried out to satisfy the eigenrelation (combining (35) and (39)):

$$
\frac{3+2 \beta}{72(1+\beta)^{2}}=\frac{1}{3} \frac{\int_{0}^{\infty}(1 / f) d \eta}{\int_{0}^{\infty}\left(1 / f^{2}\right) d \eta}
$$


These computations give a unique value $\beta=0.175$ correct to three decimals. Representative solutions are given in Figure 1 from which it is seen that the effect of $f_{2}$ is just a rescaling in $\eta$ as expected.

This Section has provided a fairly complete quantitative description of possible terminal pinching states of Stokes jets. The most notable feature of the analysis is that unique scaling exponents are fixed together with universal scaling functions to within a multiplicative constant which depends on the initial conditions. The uniqueness arises by solving an eigenvalue problem for symmetric pinching profiles. The remainder of the article is concerned with the verification of the local analysis just described by detailed comparison with numerical solutions of the model system for different initial conditions.

\section{$5 \quad$ Numerical solutions.}

Numerical solutions have been obtained on axially periodic domains which can, without loss of generality, be normalized to have length $2 \pi$. Since the pinching phenomenon is a local one the periodicity in the boundary conditions is not expected to play a fundamental role. The equations to be solved numerically are

$$
\begin{aligned}
S_{0 t}+w_{0} S_{0 z}+\frac{1}{2} w_{0 z} S_{0} & =0 \\
\left(3 S_{0}^{2} w_{0 z}+S_{0}\right)_{z} & =0 .
\end{aligned}
$$

As will be shown shortly the initial condition $S_{0}(0, z)=F(z)$ alone is required. It is clear from (41) that

$$
\frac{\partial}{\partial t}\left(\int_{0}^{2 \pi} S_{0}^{2} d z\right)=0
$$

which provides a useful conserved quantity in controlling the accuracy of the computations. Equation (42) can be integrated once with respect to $z$ to yield

$$
w_{0 z}=\frac{1}{3}\left(\frac{\lambda(t)}{S_{0}^{2}}-\frac{1}{S_{0}}\right),
$$

where $\lambda(t)$ is a function of time to be determined. Integration of (44) with respect to $z$ and use of periodicity yields a value for $\lambda(t)$ in terms of $S_{0}$ :

$$
\lambda(t)=\frac{\int_{0}^{2 \pi}\left(1 / S_{0}\right) d z}{\int_{0}^{2 \pi}\left(1 / S_{0}^{2}\right) d z} .
$$

Equation (44) combined with (45) shows that an initial condition for $S_{0}$ alone is sufficient to determine the evolution.

Spectral methods are used to solve the equations numerically, and all required integrations are also done spectrally. The result used is that the discrete fourier transform of a periodic function, $q(z)$ say, and the integral of $q(z)$ are related by the following expression which follows directly from the definition of fourier transforms:

$$
\hat{q}(k=0)=\int_{0}^{2 \pi} q(z) d z .
$$


For example $\lambda(t)$ in (45) is computed by calculating the fourier transforms of $\left(1 / S_{0}\right)$ and $\left(1 / S_{0}^{2}\right)$ and forming the ratio of the first two fourier components. With these results available the numerical strategy is as follows: Equation (41) is marched forward in time by specification of $S_{0}(0, z)$ alone; $\lambda$ is computed as indicated above at each level of a multi-level time scheme which then provides $w_{0 z}$ through (44) at that level; to update $S_{0}$ in time using (41) the function $w_{0}$ is also required. This is evaluated by transforming (44) into Fourier space by use of fast fourier transforms and inverting $(i k)^{-1} \hat{H}$, where $\hat{H}$ is the discrete fourier transform of $\frac{1}{3}\left(\frac{\lambda(t)}{S_{0}^{2}}-\frac{1}{S_{0}}\right)$. (note that the fourier transforms of $\left(1 / S_{0}\right)$ and $\left(1 / S_{0}^{2}\right)$ are already known at this stage from the computation of $\lambda$ ). Finally the remaining derivative $S_{0 z}$ in (41) is evaluated pseudospectrally and the time integration is done in real space. A second order accurate predictor-corrector scheme as well as a third order Runge-Kutta method were implemented and tested. The computations described here were generated by the second order scheme. Certain symmetries in the equations were also utilized in the numerical work and to provide numerical solutions that can be directly compared with the similarity solutions described earlier. It can be seen that if $S_{0}$ is an even function of $z$ initially, then it will remain so for subsequent times since $w_{0}$ is then odd as seen from (44). More formally it is easy to show by integration by parts of (44) that

$$
<w_{0}>=\frac{1}{3<S_{0}^{-2}>}\left(-<S_{0}^{-1}><z S_{0}^{-2}>+<S_{0}^{-2}><z S_{0}^{-1}>\right),
$$

where $\langle()\rangle=.\int_{0}^{2 \pi}() d$.$z , and from which it follows immediately that w_{0}$ is odd if $S_{0}$ is even. With $w_{0}$ odd it is ensured that its zero fourier component vanishes and does not enter into the fourier inversion procedure of its computation outlined above. For general initial conditions, however, the inversion to find $w_{0}$ can only be performed if the first fourier mode (the one corresponding to $k=0$ ) is provided. In the notation of (46) this is just $\left\langle w_{0}\right\rangle$ which is easily found by performing the additional fourier transforms of $z / S_{0}^{2}$ and $z / S_{0}$ respectively, or quadrature methods. Both were implemented and used with no noticable change in the results.

In our computations of symmetric solutions we use the following initial condition

$$
S(0, z)=a+b \cos (z) .
$$

This choice gives a minimum in the initial condition at $z=\pi(b>0)$ and symmetry about this point with pinching first taking place there. The corresponding axial velocity is then zero at $z=\pi$ and an odd function of $z-\pi$. Throughout the computation the evolution of $\left\langle S_{0}^{2}\right\rangle$ and $\left\langle w_{0}\right\rangle$ was monitored in order to confirm that the former is conserved and the latter is zero due to parity; in all the results given here these integral constraints varied only due to machine round-off errors. Besides the actual solutions at different times, it is important to monitor the evolution of $\lambda(t)$ and the value of the minimum as a function of $t$ which we denote by $S_{\min }(t)$. These quantities are crucial in our comparisons with the similarity solutions of Section 4 . The axial position where the minimum is attained was also monitored in order to verify accuracy, since the minimum is stationary for the present choice of initial conditions. We note that the accuracy tests are crucial in determining acceptable solutions which can be used to verify the asymptotic theory especially since such comparisons are only meaningful when the jet radius is very small and the equations become singular. All solutions given here pass the integral accuracy tests outlined above. 
Before presenting numerical results we consider the linear stability of the system (41) and (42) which is being solved numerically. Linearization is done about a constant value of $S_{0}$ taken to be $a$ for consistency with the initial condition and about the zero state for $w_{0}$. Linearization of (41) and (42), then, and elimination of $w_{0 z z}$ by differentiation of the first equation with respect to $z$ gives the following equation for the linear perturbation denoted by $S_{0}$ also,

$$
S_{0 z t}-\frac{1}{6 a} S_{0 z}=0
$$

The solution is readily found to be

$$
S_{0}(t, z)=S_{0}(0, z) e^{\frac{1}{6 a} t}
$$

The linear solution (49) is identical to the growth rate of the $k=0$ mode of linear theory as expected (this follows from (1) and (2) once the non-dimensionalizations (3) are introduced). It can be seen from (49) that linear perturbations grow in place, so that a depression (regions where $S_{0}(0, z)$ is negative) tends to grow reducing the local jet radius with the opposite happening in regions where there is a local elevation. Physically this implies that fluid is being pushed out of a necking region and towards bulging regions, a result which is in line with the mechanism of capillary instability since surface tension tends to increase the pressure just below the surface of a depression and decrease it near elevations, causing a fluid motion from high pressure regions to low pressure ones. ¿From a numerical point of view the linear result (49) indicates that numerical short wave round-off error disturbances which are inevitably introduced into the numerical simulation, are not subject to pathological instabilities as in Kelvin-Helmholtz or related problems (see Krasny [23] and Papageorgiou and Smith [24] for instance).

The first set of numerical experiments has an initial condition with $a=0.5$ and $b=0.1$. The number of modes used is 512 and the time-step was 0.0005 by the end of the computation. The computation was terminated when the minimum jet radius, $S_{\min }$, became smaller than 0.003 . This happened at approximately $t=6.6$. In order to achieve such a small value of $S_{\min }$ the computational step was refined during the stage $6.5<t<6.6$. Numerical convergence was checked by performing an identical computation with 256 modes; the only difference is that the higher resolution computation allows achievement of a slightly smaller minimum radius for a given accuracy. The evolution of the jet surface $S_{0}(t, z)$ and the corresponding axial velocity $w_{0}(t, z)$ are given in Figures 2(a),(b). Figure 2(a) depicts the evolution for $0<t<6.5$ while Figure 2(b) that during $6.5<t<6.6$. As the Figures indicate the jet is pinching after a finite time; the radius vanishes first at $z=\pi$. The final computed shape shown as a cross-section of the jet, along with the evolution of the maximum value of $S_{x}$ over the spatial domain, is given in Figure 3. The jet shape appears fairly flat near the pinch point (the slope is in fact zero there) and in particular the slope is bounded at all axial positions. This is an essential requirement for the validity of the long-wave model equations. The leading order axial velocity distribution has the following features: The flow is stagnant (to leading order) at $z=\pi$ where a pinch first appears. Just to the right and left of the pinch point there are large axial velocities away from the pinch points; these axial velocities become infinite in magnitude as the singular time is approached and fluid drains out of the necking region at increasingly higher local speeds. We will use the results from 
this numerical experiment to perform a detailed comparison with the asymptotic theory of Section 4, and in particular will derive the scaling exponents and the rate at which the force in the jet is going to zero as the singular time is approached.

Figure 4 shows the evolution of $S_{\min }(t)$, which is seen to approach zero after a finite time - the rate at which this is done is found to be linear (see later for an estimate of the slope), and as shown in the enlarged section the time of the singularity can be estimated by a least squares fit. The evolution of the maximum axial velocity with time, along with the corresponding evolution of the axial position where it is attained, is shown in Figure 5. These results strongly suggest that the axial velocity blows up at a finite time and this happens locally at some axial position. The rate at which the velocity blows up will be compared with the self-similar solutions later. Note that due to symmetry there is a symmetrically placed minimum in $w_{0}$ which blows up at the same rate as the maximum. Finally, in Figure 6 we depict the evolution of $\lambda(t)$ given by (45) noting that physically this quantity represents the quasi-uniform force, to leading order, throughout the jet at different times. Clearly $\lambda(t)$ is approaching zero after a finite time; in fact for times larger than approximately 4.0 the dependence of $\lambda$ with $t$ is established to be linear, a fact which is used to get an estimate of the singular time, $t_{s}$, by a least squares fit as above.

The results just presented indicate a qualitative picture of the breakup phenomenon: the radius of the jet goes to zero linearly and so does the force in the jet. At the same time the axial velocity blows up after a finite time. These numerical results are used next to make a direct comparison with the similarity solutions constructed in Section 4. Since the pinching similarity solutions of the model equations are the same as those for the Stokes equations (see Sections 4.1 and 4.2 respectively) the comparison holds for both regimes. According to the ansatz (23), then, along with the solutions (38) it is easy to determine $S_{\min }(t)$ given by the local similarity theory,

$$
S_{\min }(t)=\left(t_{s}-t\right) \frac{1}{12(1+\beta)},
$$

where $\beta=0.175$ (see Section 4.3). An expression for $\lambda(t)$ near the singular time follows similarly from the analysis of Section 4 and yields

$$
\lambda(t)=3 k\left(t_{s}-t\right)=\frac{3+2 \beta}{24(1+\beta)^{2}}\left(t_{s}-t\right),
$$

where the expression (35) has been used to express $k$ in terms of $\beta$. The analytical expressions (50) and (51) allow for a direct comparison between the theory and the numerical experiments. To carry this out, however, we require the value of the singular time, $t_{s}$. This is not provided directly by the numerical solutions but can be estimated with tolerable accuracy from the data which make up Figures 4 and 6 . A value of $t_{s}$ was obtained as follows: a least squares fit was applied to the data in Figure 4 for $t>5.0$ and extrapolation was used to find $t_{s}$ the point where the curve (straight line) intersects the $t$-axis. This was repeated with least squares fits of smaller sets of data nearer the singular time with no change in the result. As a check the $t_{s}$ estimated by least squares fits of the $\lambda(t)$ data (Figure 5 ) yields the same value of $t_{s}$ to four decimal places. This value is

$$
t_{s}=6.6405 \text {. }
$$


Using this value of $t_{s}$ we can check the power law behavior of the computed solutions near the singular time. This is done as follows: We take the sets of data $\left(t, S_{\min }(t)\right),(t, \lambda(t))$, $\left(t, w_{\max }(t)\right)$ from Figures 4,5 and 6 respectively for $t>5.9$ (this is near enough the singularity for the asymptotic structures to provide a good approximation to the exact solutions) and produce plots of the sets $\left\{\left(\log \left(t_{s}-t\right), \log \left(S_{\min }(t)\right)\right\}\right.$ and so on; the slopes of these lines should give the powers of $\tau$ appearing in the similarity ansatz (23). The results are depicted in Figure 7; the slopes are estimated by least squares fits and are correct to the number of decimal places indicated on the diagrams. The results are also summarized in Table 1 below.

\begin{tabular}{|c|c|c|}
\hline Scaling law & Asymptotic & Numerical \\
\hline \hline$S_{\min }\left(t_{s}-t\right)$ & 1.0 & 1.0 \\
\hline$\lambda\left(t_{s}-t\right)$ & 1.0 & 1.0 \\
\hline $\max \left\{w_{0}\right\}\left(t_{s}-t\right)$ & $\beta-1=-0.825$ & -0.823 \\
\hline
\end{tabular}

\section{Table 1}

The results just presented verify the power law behavior postulated by the similarity ansatz (23). The value of $\beta=0.175$ is therefore supported by the numerical solutions also, and next we make a further comparison of the multiplicative constants also. As shown above, the leading order behavior of $S_{\min }(t)$ and $\lambda(t)$ according to the asymptotic theory of Section 4 as the singular time is approached is given by (50) and (51). In Figures $8(\mathrm{a}),(\mathrm{b})$ we superimpose the numerically computed evolution of $S_{\min }$ and $\lambda$ with the corresponding asymptotic forms $(50)$ and (51). It is seen that at times larger than about 4.0 (which is still at least 2 time units from the singular time) agreement is excellent; the divergence of the two curves for smaller times is expected.

The results of Figures 3-8 provide strong evidence that the initial value problem of the model equations terminates in a singularity after a finite time according to the theoretical predictions set out in Section 4. The comparisons above were designed to verify the power law behavior of the solutions and in what follows we consider a comparison of the solutions of the model equation near the singular time with the scaling functions found from the asymptotic theory. The numerical experiment we have been concentrating on terminates at a singular time estimated to be $t_{s}=6.6405$. We describe next how to construct the scaling functions $f(\xi)$ and $g(\xi)$ from our numerical solutions near the singular time: Given data $S_{0}(t, z)$ and $w_{0}(t, z)$ it is easy to compute $\xi$ and the corresponding values of $f(\xi)$ and $g(\xi)$ by direct substitution of $\tau=\left(t_{s}-t\right)$ into the forms (23). Different scaling functions are obtained for different $\tau$ but all results should collapse to universal curves as $\tau \rightarrow 0$. This is indeed the case as depicted in Figures 9(a), (b) corresponding to $f(\xi)$ and $g(\xi)$ respectively.

\subsection{General initial conditions; non-parity solutions}

The results described so far have been computed by imposing a symmetry on the evolution equations, restricting solutions to shapes which are symmetric and axial velocities which are anti-symmetric about $z=\pi$. This was done in order to obtain an accurate set of data to use in the evaluation of the asymptotic self-similar theory. The self-similar theory, however, is a local one and it does not require the parity requirements mentioned above for all time. We 
would expect locally symmetric solutions, then, to appear as correct leading order dynamics in the breakup described by the model equations (41) and (42) starting from general initial conditions which do not ensure parity for subsequent times. Equations (41) and (42) were solved numerically (see earlier) using $n=512$ modes and the initial condition

$$
u_{0}(x)=0.5+0.1(\sin (x)+\cos (x)) .
$$

The evolution up to $t=5.0$ of the interfacial shape and the corresponding axial velocity is given in Figure 10. Due to the loss of symmetry, the jet first breaks at a point not equal to $\pi$. The results of this numerical experiment are used next to confirm the local validity of the self-similar solutions. In Figure 11 we give the evolution of $S_{\min }(t)$ and $\lambda(t)$ up to the time when $S_{\min }$ is less than 0.005 . Superimposed on these curves are the results given by the asymptotic forms (50) and (51) with $\beta$ determined by the similarity solutions $(\beta=0.175)$ and $t_{s}$ determined numerically from the data by first confirming that the curves are straight lines as $t \rightarrow t_{s}$, followed by extrapolation to obtain $t_{s}$. It is seen that agreement is excellent. A more severe test is a direct numerical check of local symmetry. This is done as follows: The profile (jet shape) is taken from the last computed time station and the minimum point on the curve is located; this gives the axial position where the jet radius is at its smallest; if we denote this position by $x_{0}$, our objetctive is to show that the jet shape is symmetric (at least locally) about $x_{0}$. A good way to see this graphically is to plot the jet shape for $x \geq x_{0}$ and superimposed onto this to plot the shape computed for $x<x_{0}$ but reflected about the line $x=x_{0}$. If there is local symmetry about $x=x_{0}$ then the two curves will coincide for a range of axial positions in the neighborhood of $x_{0}$. The results are shown in Figure 12(a) with the circles denoting the reflected shape. We see that the two curves are indistinguishable for a large range of values about $x_{0}$ and so we conclude that the solution at pinching is locally symmetric. The corresponding axial velocity distribution becomes asymetric as a pinch is formed, and the confirmation of this based on our numerical results is given in Figure $12(\mathrm{~b})$ which depicts plots of $\left(x, w_{0}(x)\right)$ for $x \geq x_{0}$ plotted along with $\left(x,-w_{0}\left(x_{0}-x\right)\right)$. Again the curves are indistinguishable confirming the local self-similar theory. Our numerical solutions, therefore, provide strong evidence that the local self-similar solutions described in Section 4 are robust in that they provide a local theory with symmetry independent of initial conditions. The initial conditions affect two things, however: (i) The singular time, and, (ii) the scaling function (and thus the final shape) at breakup. These two features are studied in more detail next by carrying out a parameter study as the initial condition varies.

\section{Breakup times and scaling functions for different initial conditions.}

It is expected that different initial conditions provide different breakup times. For example, it would be expected that the breakup time decreases as the minimum initial jet radius is decreased. In what follows we try to quantify such statements by carrying out a series of numerical experiments - this is done for both symmetric and asymmetric solutions. Before presenting results we define what we mean by breakup time. All runs (unless otherwise stated) are followed up to times when the minimum jet radius first becomes less than 0.005 . 
The evolution of $\lambda(t)$ is then used to obtain an estimate of the singular time by extrapolation after utilizing the linear form of $\lambda(t)$ near the singular time (see Section 5). The self-similar shapes at breakup are then approximated by the data from the last computed time, after applying the transformations of (23).

One aim of carrying out such extensive computations is in the evaluation of linear stability theory, estimates of the breakup time for instance, as compared to the nonlinear dynamics. Working within the framework of the one-dimensional model, we can use linear theory to obtain an empirical estimate of the breakup time. As shown in (49) linear solutions grow exponentially at a rate $\exp (t / 6 a)$; the total solution which results from an initial condition $h(z)$ is

$$
S(z, t)=a+\delta h(z) \exp (t / 6 a)
$$

where $\delta$ is the infinitessimally small amplitude of the perturbation as is usual in linear theories. Denoting the minimum of $h(z)$ over the domain by $-h_{0}<0$, we can use (53) to predict a time, $t_{L}$ say, when $S\left(z, t_{L}\right)$ first becomes zero. This is easily calculated to be

$$
t_{L}=6 a \ln \left(\frac{a}{\delta h_{0}}\right),
$$

and represents the breakup time as predicted by linear theory. We emphasize that this calculation is empirical in that, for example, $t_{L}$ is a time outside the range of validity of linear theory (linear theory is valid when $S(z, t)$ is near $r=a$ ). Similar ideas have been used in [25] in describing the rupture of free viscous films in the presence of van der Waals forces.

\subsection{Symmetric initial conditions}

Symmetry in the jet shape is preserved throughout the evolution if the initial condition is symmetric. In the results that follow we computed breakup times as a function of an initial amplitude $\epsilon_{1}$ where

$$
S_{0}(z, 0)=0.5+\epsilon_{1} \cos (x) .
$$

The parameter $\epsilon_{1}$ is also a measure of the initial energy provided by the disturbance. We note that $\epsilon_{1}<0.5$, otherwise the initial condition has zero radius to start with - the breakup time in this case is defined to be zero. The results are summarized in Figure 13 which depicts the variation of the breakup time, $t_{s}$, with $\epsilon_{1}$. The figure also contains the breakup time $t_{L}$ predicted by the linear result (54), noting that in this case $h_{0}=1$. It is seen that agreement between the two is only reasonable when $\epsilon_{1}$ is small as would be expected (we emphasize once more, however, that the linear estimate albeit empirical can be useful in some instances). For example, an initial perturbation $\epsilon_{1}=0.05$ (a $10 \%$ disturbance of the unperturbed jet radius) gives a breakup time $t_{s}=9.04$ while linear theory predicts $t_{L}=6.91$, an underestimate of approximately $26 \%$. At a $20 \%$ initial perturbation the error is approximately $27 \%$. An additional feature of the numerical results, which is in line with intuition, is that the breakup time decreases as the initial amplitude increases. In fact for initial amplitudes larger than about 0.2 (i.e. a $40 \%$ perturbation of the unperturbed radius) the breakup time varies almost linearly with $\epsilon_{1}$. The numerical values that make up Figure 13 are also given in Table 2 below. 


\begin{tabular}{|c|c|c|c|c|c|c|c|c|c|c|c|c|c|}
\hline$\epsilon_{1}$ & .001 & .005 & .01 & & 02 & .03 & .04 & .05 & .075 & .1 & .125 & .15 & . .175. \\
\hline$t_{s}$ & 21.13 & 16.28 & 14.1 & & .00 & 10.71 & 9.78 & 9.04 & 7.66 & 6.64 & 5.82 & 5.13 & 4.53 \\
\hline$t_{L}$ & 18.64 & 13.82 & 11. & & 66 & 8.44 & 7.58 & 6.91 & 5.69 & 4.83 & 4.16 & 3.61 & 3.15 \\
\hline$\epsilon_{1}$ & .2 & .225 & .25 & .275 & .3 & .325 & .35 & .375 & .4 & .425 & .45 & .475 & .5 \\
\hline$t_{s}$ & 4.00 & 3.52 & 3.08 & 2.68 & 2.31 & 1.95 & 1.63 & 1.32 & 1.03 & .751 & .487 & .230 & 0 \\
\hline$t_{L}$ & 2.75 & 2.40 & 2.08 & 1.79 & 1.53 & 1.29 & 1.07 & 0.86 & 0.67 & 0.49 & 0.32 & 0.15 & 0.0 \\
\hline
\end{tabular}

\section{Table 2}

\subsection{Non-symmetric initial conditions}

The results that follow were generated from an initial condition of the form

$$
S_{0}(z, 0)=0.5+\epsilon_{1}(\sin (x)+\cos (x)) .
$$

Noting that this can be re-written as

$$
S_{0}(z, 0)=0.5+\sqrt{2} \epsilon_{1} \cos (x-\pi / 4),
$$

we see that the value $\epsilon_{1}=\sqrt{2} / 4 \approx 0.35355$ is an upper bound for the initial perturbation amplitude. The breakup times were computed as before, and the estimate $t_{L}$ provided by equation (54) is valid with $\delta=\sqrt{2} \epsilon_{1}$ now. Figure 14 shows the variation of the computed breakup time with the initial amplitude level $\epsilon_{1}$, together with the linear result $t_{L}$. Table 3

\begin{tabular}{|c|c|c|c|c|c|c|c|c|c|c|c|}
\hline$\epsilon_{1}$ & .001 & .00 & & .0 & & .02 & .03 & .04 & .05 & .075 & .1 \\
\hline$t_{s}$ & 20.09 & 15. & & 13. & & 10.90 & 9.59 & 8.63 & 7.86 & 6.43 & 5.36 \\
\hline$t_{L}$ & 17.60 & 12. & & 10. & & 8.62 & 7.40 & 6.54 & 5.87 & 4.65 & 3.79 \\
\hline$\epsilon_{1}$ & .15 & .2 & .2 & & .275 & .3 & .31 & .32 & .33 & .34 & .35 \\
\hline$t_{s}$ & 3.76 & 2.56 & 1.5 & & 1.16 & .76 & .61 & .46 & .32 & .18 & .05 \\
\hline$t_{L}$ & 2.57 & 1.71 & 1.0 & & 0.75 & 0.49 & 0.39 & 0.30 & 0.21 & 0.12 & 0.03 \\
\hline
\end{tabular}
below provides the numerical values that comprise the figure.

\section{Table 3}

Qualitatively, then, the behavior is similar to the symmetric case. A more direct comparison between the two types of numerical experiment is made in Figure 15. Here the breakup times are plotted as a function of the energy of the initial perurbation defined by

$$
E=\frac{1}{2 \pi} \int_{0}^{2 \pi}\left(\left(S_{0}(z, 0)\right)^{2}-a^{2}\right) d z .
$$

Applying this to the initial conditions used above gives the following expressions for the perturbation energy corresponding to symmetric, $E_{s}$ say, and asymetric, $E_{a}$ say, initial conditions,

$$
E_{s}=\frac{\epsilon_{1}^{2}}{2}, \quad E_{a}=\epsilon_{1}^{2} .
$$

Figure 15 shows collectively the breakup times as a function of $E_{s}$ and $E_{a}$. It can be concluded from these results that the jet breaks sooner for the case of asymmetric initial 
conditions; for the particular choice of initial conditions used here the breakup times for the symmetric case are always larger than the corresponding ones for asymmetric conditions, at a given equal initial perturbation energy. Asymmetry appears to enhance breakup, then.

\subsection{Scaling functions for different initial conditions}

Here we construct the behavior of the solutions near the singular time for different initial conditions. For brevity we consider pinching solutions computed from symmetric initial interfacial elevations - non-symmetric conditions are treated in the same way and have already been shown to provide locally symmetric solutions at breakup (see Figure 12). The main objective of this section is to provide numerical evidence that different initial conditions will pinch according to a single scaling function when suitably normalized. We constructed scaling functions near pinching by solving the initial value problem for different $\epsilon_{1}$ in (6.1). The scaling functions were constructed as described earlier (for instance the methods used in the construction of Figure 9) and by use of the computed estimates of singular times from Table 2. In what follows we concentrate on the interfacial shape scaling functions derived from numerical solutions starting from initial conditions with $\epsilon_{1}=0.005,0.1,0.2,0.3$. According to the similarity solutions of Section 4 , all scaling functions coincide at $\xi=0$ where they take the value $1 /(12(1+\beta))$. This will be exhibited in the construction of the scaling functions below, but can also be seen by consideration of the variation of $u_{\min }(t)$ as the singular time is approached - the variation of $u_{\min }(t)$ with $t$ near the singular time $t_{s}$ for a given initial condition, should be linear with slope $1 /(12(1+\beta)$ ) (see $(50))$. Figure 16 provides the variation of $u_{\text {min }}(t)$ for different initial conditions $\epsilon_{1}=0.005,0.01,0.02,0.03,0.05,0.1,0.2,0.3$ labeled on the figure and superimposed with these numerical results is the asymptotic behavior near the singular time (see above and equation (50)) shown with open circles. It is seen that agreement is achieved near the singular times as expected.

The scaling functions for different initial conditions are considered next. As shown by the results of Figure 16, all scaling functions are equal to the universal constant $1 /(12(1+$ $\beta) \approx 0.0709$ at $\xi=0$. Numerically constructed scaling functions from initial conditions characterized by the amplitudes $\epsilon_{1}=0.005,0.1,0.2,0.3$ are shown in Figure 17(a) which indicates the self-similar nature of the different terminal scaling functions. It can be seen from the analytical solution (38) that the only difference between scaling functions is expected to appear through the constant $A$ in (38) which in turn depends on initial conditions; the role of this constant is to stretch the axial coordinate $\eta$ by different amounts for different initial conditions. Our numerical simulations and singular states fully support this property of the solutions as is demonstrated next: Choose the computed scaling function corresponding to $\epsilon_{1}=0.1$ as the reference function, $f_{0}(\eta)$ say. According to the theory, for each of the other initial conditions shown in Figure 17(a), a number, $c$ say, can be found so that the change of variables $\eta \rightarrow c \eta$ maps the given scaling function into the normalizing one. The number $c$ is different for different initial conditions and was computed by calculating the ratio $\eta_{0} / \eta_{i}$ where $f_{0}\left(\eta_{0}\right)=f_{i}\left(\eta_{i}\right)=h$ with $h$ a fixed interfacial amplitude-different values of $h$ produce the same ratios as expected (the value of $h$ had to be found by interpolation due to the nonuniformity of the grids). This procedure, then, enables all scaling functions to be collapsed onto $f_{0}(\eta)$. The result of this calculation is given in Figure $17(\mathrm{~b})$ which provides additional evidence for the validity of the asymptotic theory near the singular time. 


\section{Computation of satellite drops}

In previous Sections we provided numerical and analytical evidence of the form of pinching solutions admitted by the model evolution equations. Even though the equations are relatively simple compared with the full governing system (the Stokes equations plus nonlinear interfacial conditions) it has been established that the model system captures a lot of the nonlinear stages of the evolution leading to pinching. In this Section we use the model to compute final breakup states which are remeniscent of the observed phenomenon of mother and satellite drop formation. To compute such solutions the initial conditions need to be chosen appropriately. In order to illustrate things we concentrate on symmetric solutions, and in particular we use a one-parameter family of initial conditions given by

$$
S_{0}(z, 0)=0.3+0.05 \cos (z)+\epsilon_{2} \exp \left(-(\pi-z)^{2}\right) .
$$

This initial condition is symmetric, periodic in $z$ and has a localized hump at $z=\pi$ over the longer wavelength depression provided by the $\cos (z)$ perturbation. The advantage of such an initial condition is that the jet pinches after a finite time with the radius vanishing simultaneously at two distinct axial positions. Just beyound pinching, then, a system of mother and satellite drops emerges. Sample results are depicted in Figure 18 for five successively increasing values of $\epsilon_{2}$. The Figure shows the cross section of the jet just before pinching; the computational domain is $2 \pi$-periodic in the axial direction and for better visualization the solution has been dipicted to include four periods. We have used the computational methods described in earlier methods to verify that the pinching takes place according to the self-similar solutions given in Section 4, and we have computed singular times as well as the volume of the satellite drops. These are estimated by evaluating the integral

$$
V_{s}=\pi \int_{z-}^{z+} S_{0}^{2} d z
$$

where $z-$ and $z+$ are the minima in $S_{0}$ to the left and right of the satellite drop respectively. The integral $V_{0}=\pi \int_{0}^{2 \pi} S_{0}^{2} d z$ is a conserved quantity which is equal to the total volume of the jet at $t=0$. The ratio $V_{s} / V_{0}$ provides a measure of the size of the drops which form after pinching. The present numerical experiments yield the results given below in Table 4, and summarized in Figure 19.

\begin{tabular}{|c||c|c|c|c|c|}
\hline$\epsilon_{2}$ & 0.03 & 0.035 & 0.04 & 0.045 & 0.05 \\
\hline$V_{s}$ & 0.229 & 0.323 & 0.393 & 0.451 & 0.502 \\
\hline$V_{0}$ & 0.602 & 0.607 & 0.612 & 0.618 & 0.623 \\
\hline$V_{s} / V_{0}$ & 0.382 & 0.532 & 0.642 & 0.730 & 0.806 \\
\hline$t_{s}$ & 7.53 & 7.22 & 7.08 & 6.96 & 6.88 \\
\hline
\end{tabular}

\section{Table 4}

It can be seen from the results that relatively small changes in the initial conditions can lead to large changes in the final drop size. Note that the initial volume of the jet increases by approximately $3 \%$ when $\epsilon_{2}$ is increased from 0.03 to 0.05 , while the volume of the satellite drop formed increases by more than $100 \%$. It appears, then, that small increases in $\epsilon_{2}$ enable 
the satellite drop to drain fluid from the adjoining main jet yielding a wide range of drop sizes. At the same time the jet breaks sooner as seen from the variation of $t_{s}$. The trends depicted in Figure 19 will be studied in more detail by more extensive computational searches in the phase space of initial conditions.

\section{Conclusions}

An asymptotic theory has been used to derive a system of nonlinear evolution equations to model the dynamics of viscous fluid jets under the action of capillary forces. The theory is a long wave expansion which allows for fully nonlinear interfacial amplitudes and the equations can predict pinching. Theoretical descriptions of the pinching have been given by use of similarity solutions valid as the jet radius tends to zero after a finite time. The scaling exponents are unique and the scaling functions form a one parameter family of similarity solutions depending on the initial conditions. The predictions of the asymptotic solutions near pinching have been confirmed, with excellent agreement, by extensive direct simulations of the initial boundary value problem. The solutions found here are possible terminal local states of the full Stokes equations and can be used to benchmark the accuracy of direct simulations (e.g. boundary integral or boundary element techniques), as well as to provide correct initial conditions to continue the computations beyond pinching. Such analyses have been described elsewhere for viscous jets (see Papageorgiou [26]). These comments are also relevant to viscous jets possessing inertia and modelled by an analogous system of evolution equations (see Appendix A).

In addition, it has been shown numerically that the model can produce pinching solutions with the radius of the jet vanishing at the same time at two distinct axial locations; this heralds the formation of a mother-satellite drop system and the model has been used to gain quantitative information on the distribution of satellite drop volumes as a function of initial conditions (or equivalently initial perturbation energy). A considerable amount of numerical experiments remain to be carried out in order to obtain overall trends in both the Stokes and Navier-Stokes models. 


\section{Appendices}

\section{A Navier-Stokes regimes}

Using the capillary scales (3) for dimensionless quantities and starting from the Navier-Stokes equations one is led to the following system:

$$
\begin{array}{r}
R_{e}\left(u_{t}+u u_{r}+u w_{z}\right)=-p_{r}+\Delta u-\frac{u}{r^{2}}, \\
R_{e}\left(w_{t}+u w_{r}+w w_{z}\right)=-p_{z}+\Delta w, \\
\frac{1}{r}(r u)_{r}+w_{z}=0,
\end{array}
$$

where the Reynolds number $R_{e}=\frac{\sigma \rho R}{\mu^{2}}\left(1 / R_{e}\right.$ is the familiar capillary number $)$. The boundary interfacial conditions are the same as (8)-(10). Proceeding as in Section 3 we introduce a long axial length-sacle by the transformation $\frac{\partial}{\partial z} \rightarrow \epsilon \frac{\partial}{\partial z}$. Our objective is to find an asymptotic solution in powers of $\epsilon^{2}$ which includes, to leading order, the effects of unsteadiness and nonlinearity in the bulk motion. In particular we wish to increase $R_{e}$ from its zero value for Stokes flows, to a value when unsteadiness and nonlinearity first enter to yield a canonical evolution system. The appropriate expansions for the flow parameters are those of Section 3 , see equations (11)-(14), together with the scale

$$
R_{e}=\epsilon^{2} \kappa, \quad \kappa=O(1) .
$$

The axial momentum equation (57) gives to the first two orders

$$
\begin{array}{r}
w_{0} \equiv w_{0}(z, t), \\
\kappa\left(w_{0 t}+w_{0} w_{0 z}\right)=-p_{0 z}+w_{1 r r}+\frac{1}{r} w_{1 r}+w_{0 z z},
\end{array}
$$

while the radial momentum equation and the continuity equation give, to leading order,

$$
\begin{array}{r}
-p_{0 r}+u_{0 r r}+\frac{1}{r} u_{0 r}-\frac{u_{0}}{r^{2}}=0, \\
u_{0}=-\frac{1}{2} r w_{0 z} .
\end{array}
$$

It follows by substitution of (62) into (61) that

$$
p_{0 r}=0 \quad \Rightarrow \quad p_{0} \equiv p_{0}(z, t) .
$$

This enables the calculation of $p_{0}$ from leading order terms in the normal stress balance condition (9), and yields

$$
p_{0}=\frac{1}{S_{0}}-w_{0 z}
$$

We are now in a position to derive the first evolution equation from the tangential stress balance equation (8). This is done as follows: The velocity correction $w_{1}$ is easily evaluated 
from (60) since the forcings $w_{0}$ and $p_{0}$ are independent of $r$. This solution, along with the expression (62) for $u_{0}$ are substituted into (8) to yield the following leading order contributions after evaluation on $r=S_{0}(z, t)$ :

$$
\begin{aligned}
w_{0 r} & =0 \\
-\frac{1}{2} S_{0} w_{0 z z}+\frac{1}{2} S_{0}\left[\kappa\left(w_{0 t}+w_{0} w_{0 z}\right)-2 w_{0 z z}+\left(1 / S_{0}\right)_{z}\right]-3 S_{0 z} w_{0 z} & =0
\end{aligned}
$$

A second evolution equation comes from the leading order contribution of the kinematic condition (10). Re-arrangement of (65) together with the kinematic contribution gives the following coupled system governing the evolution of $w_{0}$ and $S_{0}$,

$$
\begin{array}{r}
\kappa\left(w_{0 t}+w_{0} w_{0 z}\right)=\frac{3\left(S_{0}^{2} w_{0 z}\right)_{z}}{S_{0}^{2}}-\left(\frac{1}{S_{0}}\right)_{z} \\
S_{0 t}+\frac{1}{2} S_{0} w_{0 z}+w_{0} S_{0 z}=0 .
\end{array}
$$

The order one parameter $\kappa$ can be scaled out of the problem by a change of variables and so we can take $\kappa \equiv 1$.

Equations (66) and (67) have been derived by Eggers and Dupont [17] by using a Taylor expansion in the $r$ coordinate, and by the present approach by Papageorgiou [26] in a slightly different regime. The present approach is a systematic single parameter asymptotic expansion with all terms appearing in the equations being of the same order in $\epsilon$. Equations (66) and (67) admit pinching similarity solutions (see [18], [19] and [26]) of the form

$$
S_{0}=\left(t_{s}-t\right) f(\xi), \quad w_{0}=\left(t_{s}-t\right)^{-1 / 2} g(\xi), \quad \xi=\frac{z}{\left(t_{s}-t\right)^{1 / 2}},
$$

where $t_{s}>0$ is the time of pinching. The scaling functions appearing in (68) satisfy

$$
\begin{array}{r}
3 g^{\prime \prime}+\frac{f^{\prime}}{f^{2}}+6 \frac{g^{\prime} f^{\prime}}{f}=\frac{1}{2}\left(g+\xi g^{\prime}\right)+g g^{\prime} \\
(g+\xi / 2) f^{\prime}-\left(1-g^{\prime} / 2\right) f=0
\end{array}
$$

with primes denoting $\xi$-derivatives. Eggers [18] and [19] has given numerical solutions of these scaling functions which do not depend on arbitrary constants and so describe pinching states which are independent of initial conditions. We note also that the system (69) and (70) arises by looking directly for pinching solutions of the Navier-Stokes equations without first deriving the intermediate asymptotic equations by introduction of $\epsilon$. Instead, the small parameter which allows for an asymptotic development is the smallness of $t_{s}-t$ near the time of pinching. This analysis (as well as the equivalent one for Stokes flows) has been carried out by Papageorgiou [15] where the dynamics beyond pinching where also modelled by use of overall mass and momentum balances. Further numerical studies of (66) and (67) are under way to evaluate the model for such phenomena as satellite drop formation and comparisons with empirical breakup times obtained from linear theory, as well as direct comparisons with experiments. 


\section{Acknowledgments}

I would like to thank Professor Charles Maldarelli of the Levich Institute for some useful discussions. 


\section{List of Figures}

Figure 1 Solutions of the similarity equations for different values of $f_{2}$ shown on the figure; left - interfacial shape, right - axial velocity.

Figure 2 Solution of the evolution equations for symmetric initial conditions: (a) $0 \leq$ $t \leq 6.5$, (b) $6.5 \leq t \leq 6.6$. The computations were stopped when $S_{\min }<0.003$.

Figure 3 (a) The final computed jet shape at $t=6.6$ when $S_{\min }=.0028$; the figure represents a cross-section of the jet in a plane containing the jet axis. (b) Evolution of the largest magnitude of the interfacial slope - boundedness of this is crucial for the long wave/slender jet theory to be valid.

Figure 4 Evolution of the minimum jet radius for symmetric initial conditions. The enlargement shows the linearity as $t \rightarrow t_{s}$ and the extrapolation to compute an estimate for $t_{s}$.

Figure 5 Symmetric initial conditions. (a) Evolution of the maximum axial velocity. (b) Evolution of the axial position where the axial velocity attains its maximum.

Figure 6 Symmetric initial conditions. Evolution of $\lambda(t)$ (cf equation (45)), the leading order component of the force in the jet. The enlargement shows the linearity as $t \rightarrow t_{s}$.

Figure 7 Symmetric initial conditions. Log-log plots estimating the power law behavior of (a) $S_{\min }(t)$, (b) $\lambda(t)$, and, (c) $w_{0 \max }(t)$, as $t \rightarrow t_{s}$.

Figure 8 Symmetric initial conditions. Comparison of the similarity solutions with the computed ones. (a) $S_{\min }(t)$, (b) $\lambda(t)$. The curves labeled asymptotic correspond to the theoretical predictions.

Figure 9 Symmetric initial conditions. Convergence to scaling functions. (a) Interfacial shape in the pinch region, (b) Axial velocity in the pinch region.

Figure 10 Solution of the evolution equations for asymmetric initial conditions. (a) Evolution of the jet shape, (b) evolution of the axial velocity.

Figure 11 Asymmetric initial conditions. Evolution of $S_{\min }(t),(\mathrm{a})$, and $\lambda(t)$, (b), together with theoretical predictions.

Figure 12 Asymmetric initial conditions. Verification of the symmetry of the interface, (a), and the asymmetry of the axial velocity, (b), near the pinch point. The solid lines denote the solution to the right of the pinch point, and the circles denote that to the left, after reflection about the pinch point.

Figure 13 Symmetric initial conditions. Breakup times for different initial amplitudes. o - numerical calculation, ${ }^{*}$ - prediction of linear theory.

Figure 14 Asymmetric initial conditions. Breakup times for different initial amplitudes. o - numerical calculation, * - prediction of linear theory.

Figure 15 Breakup times for symmetric and asymmetric initial conditions plotted as functions of the initial perturbation energy. o - symmetric, ${ }^{*}$ - asymmetric.

Figure 16 Variation of $S_{\min }(t)$ for different symmetric initial conditions, $\epsilon_{1}=0.005$, $0.01,0.02,0.03,0.05,0.1,0.2,0.3$. The open circles show the asymptotic result according to equation $(50)$ and the magnitude of their slopes is equal to $1 /(12(1+\beta))$.

Figure 17 Symmetric initial conditions. The interfacial scaling functions near pinching for different initial conditions. (a) Non-normalized, (b) normalized with the scaling function corresponding to $\epsilon_{1}=0.1$. 
Figure 18 Runs leading to satellatite drop formation. Numerical solutions generated for different initial conditions $(55)$ and $\epsilon_{2}=0.03,0.035,0.04,0.045,0.05$ shown on the Figure. A jet cross section is shown just before breakup.

Figure 19 Satellite drop formation. (a) Variation of the ratio of satellite drop volume to total jet volume with initial conditions; (b) Corresponding jet breakup times. 


\section{References}

[1] Lord Rayleigh, "On the stability of liquid jets", Proc. Lond. Math. Soc. 10, 4-13 (1878).

[2] Lord Rayleigh, "On the stability of a cylinder of viscous liquid under capillary force", Scientific Papers, iii, 585-93, Cambridge, England, (1902). (also Phil. Mag., 34, p. 145 $(1892))$

[3] S. Tomotika, "On the stability of a cylindrical thread of a viscous liquid surrounded by another viscous fluid", Proc. R. Soc. Lond. A150, 322-337 (1935).

[4] S. Chandrasekhar, "Hydrodynamics and Hydromagnetic Stability", Oxford: Clarendon Press (1961).

[5] K.C. Chaudhary and T. Maxworthy, "The nonlinear capillary instability of a liquid jet. Part 2. Experiments on jet behavior before droplet formation", J. Fluid Mech. 96, $275-286$ (1980).

[6] K.C. Chaudhary and T. Maxworthy, "The nonlinear capillary instability of a liquid jet. Part 3. Experiments on satellite drop formation and control", J. Fluid Mech. 96, $287-297$ (1980).

[7] R.J. Donelly and W. Glaberson, "Experiments on the capillary instability of a liquid jet", Proc. Roy. Soc. Lond. A290, 547-556 (1966).

[8] E.F. Goedde and M.C. Yuen, "Experiments on liquid jet instability", J. Fluid Mech. 40, 495-511 (1970).

[9] D.H. Peregrine, G. Shoker and A. Symon, "The bifurcation of liquid bridges", J. Fluid Mech. 212, 25-39 (1990).

[10] M. Tjahjadi, H.A. Stone and J.M. Ottino, "Satellite and subsatellite formation in capillary breakup", J. Fluid Mech. 243, 297-317 (1992).

[11] H.A. Stone and L.G. Leal, "Relaxation and breakup of an initiallly extended drop in an otherwise quiescent fluid", J. Fluid Mech. 198, 399-427 (1989).

[12] J.R. Richards, A.M. Lenhoff and A.N. Beris, "Dynamic breakup of liquid-liquid jets", Phys. Fluids 6(8), 2640-2655 (1994).

[13] D.B. Bogy, "Drop formation in a circular liquid jet", Ann. Rev. Fluid Mech. 11, 207-228 (1979).

[14] L. Ting and J.B. Keller, "Slender jets and thin sheets with surface tension", SIAM J. Appl. Math. 50 No. 6, 1533-1546 (1990).

[15] D.T. Papageorgiou, "Analytical description of the breakup of liquid jets", submitted to J. Fluid Mech. (1994). 
[16] M. Renardy, "Some comments on the surface-tension driven breakup (or lack of it) of viscoelastic jets", J. Non-Newtonian Fluid Mech. 51, 97-107 (1994).

[17] J. Eggers and T.F. Dupont, "Dropformation in a one-dimensional approximation of the Navier-Stokes equation", J. Fluid Mech. 262, 205 (1994).

[18] J. Eggers, "Universal pinching of 3D axisymmetric free-surface flow", Phys. Rev. Lett. 71, 3458-3460 (1993).

[19] J. Eggers, "Theory of drop formation”, Preprint (1994).

[20] F.J. García and A. Castellanos, "One-dimensional models for slender axisymmetric viscous liquid jets", Phys. Fluids 6(8), 2676-2689 (1994).

[21] W.W. Schultz and S.H. Davis, "One-dimensional liquid fibers", J. Rheol. 26(4), 331-345 (1982).

[22] M.M. Denn, "Drawing of liquids to form fibers", Ann. Rev. Fluid Mech. 12, 365-387 (1980).

[23] R. Krasny, "A study of singularity formation in a vortex sheets by the point vortex approximation”, J. Fluid Mech. 167, 65-93 (1986).

[24] D.T. Papageorgiou and F.T. Smith, "Nonlinear stability of the wake behind a flat plate placed parallel to a uniform stream", Proc. R. Soc. Lond. A 419, 1-28 (1988).

[25] T. Erneux and S.H. Davis, "Nonlinear rupture of free films", Phys. Fluids A 5(5), 1117-1122 (1993).

[26] D.T. Papageorgiou, "Breakup of liquid jets governed by the Navier-Stokes equations", ICASE/LaRC Interdisciplinary Series in Science and Engineering, Transition, Turbulence and Combustion, Vol. I, Transition, eds M.Y. Hussaini, T.B. Gatski and T.L. Jackson, Kluwer Academic Publishers, (1994). 